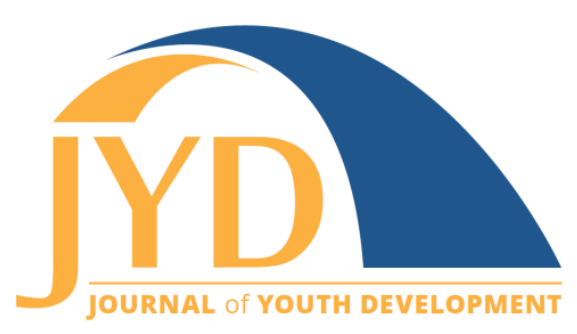

http://jyd.pitt.edu/ | Vol. 15 Issue 4 DOI 10.5195/jyd.2020.879 | ISSN 2325-4017 (online)

\title{
It's About Time: Staff Support Contacts and Mentor Volunteer Experiences
}

\author{
Thomas E. Keller \\ Portland State University \\ kellert@pdx.edu
}

\begin{abstract}
Alison L. Drew
Boston University

adrew@bu.edu
\end{abstract}

Hyuny Clark-Shim

Portland State University

h.clarkshim@pdx.edu

\section{Renée Spencer}

Boston University

rspenc@bu.edu

\section{Carla Herrera}

Herrera Consulting Group, LLC

carlaherrera66@gmail.com

\begin{abstract}
Formal youth mentoring programs typically rely on volunteers to serve as mentors to young people, with training and guidance from agency staff. A fundamental program practice is to provide ongoing support and supervision to volunteer mentors by engaging in regular contact to monitor the progress of the mentoring relationship and offer guidance and encouragement. Using data from mentors $(\mathrm{n}=504)$ in multiple mentoring programs ( $\mathrm{n}=55)$, the current study investigated how the amount of time devoted to these match support contacts was associated with mentor perceptions regarding the nature of their volunteer experience, specifically: the quality of supervision received, the mentoring agency's organizational culture with respect to engaging volunteers, and satisfaction with their volunteer service experience. Mentors who had no staff support contacts gave the lowest ratings for quality of supervision, organizational culture, and service experience. Further, mentors who typically had the shortest support contacts (1 to 5 minutes) reported lower quality supervision, organizational culture, and service
\end{abstract}

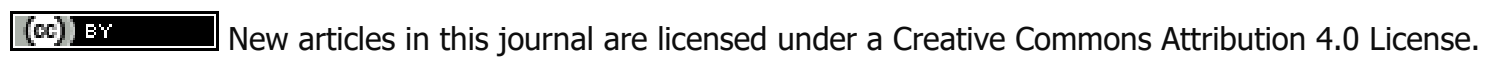
This journal is published by the University Library System, University of Pittsburgh and is cosponsored by the University of Pittsburgh Press. The Journal of Youth Development is the official peer-reviewed publication of the National Association of Extension 4-H Youth Development Professionals and the National AfterSchool Association. 


\section{Support Contacts for Volunteer Mentors}

experience compared to mentors with longer support contacts. In most cases, the associations between staff support contact length and mentor-reported outcomes remained significant after controlling for the helpfulness of the support contacts and the frequency of using advice suggested during the contacts. These findings provide evidence that match support contacts are an important practice for youth mentoring programs and suggest that programs should encourage staff to spend time engaging in meaningful conversations beyond quick check-ins. Future research should examine how the content of support contacts influences volunteer mentoring experiences.

Key words: youth mentoring, match support, program support, volunteer supervision, organizational culture

Formal youth mentoring programs typically rely on volunteers from the community to serve as mentors to young people. Despite declining volunteerism in the United States overall, the rate at which people volunteer to be mentors has increased modestly over time, with approximately 2.5 million adults serving in this capacity (Raposa et al., 2017). Mentoring programs devote considerable effort to recruiting volunteers, but retaining those volunteers is critical for mentoring relationships to last long enough to have positive effects on youth development (Grossman et al., 2012; Grossman \& Rhodes, 2002). Features of the mentoring program that influence the volunteer experience can be important factors in volunteer retention (Stukas et al., 2014). Research on volunteering suggests that program experiences that align with volunteer motivations, meet volunteer goals, and promote positive feelings about involvement with the organization result in higher satisfaction and greater retention (Clary et al., 1998; Omoto \& Snyder, 1995; Penner, 2002). Likewise, experiences that foster the development of role identity as a volunteer of a particular organization also encourage continued commitment (Grube \& Piliavin, 2000).

Most formal youth mentoring programs have a two-tiered structure in which program staff supervise and support the volunteer mentors who interact directly with the youth mentees (Garringer et al., 2017). This structure reflects a service delivery or workplace model of volunteer involvement, in which most of the work of the organization is performed by volunteers who are recruited, trained, and supervised for their specific role by paid staff members (Rochester, 1999). In terms of program operations, this type of traditional, hierarchical structure favors established roles and responsibilities and the application of standards and best practices in the management of volunteers (Macduff et al., 2009). In Rehnborg's (2009) framework for classifying volunteer roles by level of specialized skill required, level of time committed, and level of affiliation to the program mission expected, mentoring typically would fall into the category of "long-term generalist" based on program expectations of volunteers from a variety of backgrounds who commit to mentoring over time. Staff management approaches important for meeting the needs of long-term generalist volunteers 
include conveying a clear vision of the program's goals, employing strong interpersonal skills, and devoting ample time to volunteers (Brudney \& Meijs, 2014).

The manner in which organizations support volunteers in learning their roles, internalizing organizational values, and developing competencies is important for establishing and sustaining effective volunteer engagement (Haski-Leventhal \& Bargal, 2008). Volunteers who feel integrated with the organizational culture, as reflected through positive relations with staff and an understanding of program goals, are more likely to express intentions to continue volunteering (Hidalgo \& Moreno, 2009). Likewise, volunteer perceptions of organizational support and positive organizational climate are associated with greater volunteer engagement, satisfaction, and commitment (Malinen \& Harju, 2017; Nencini et al., 2016).

In the youth mentoring intervention, the interpersonal interactions between program staff and volunteers represent the "point-of-service" processes through which program goals, policies, and procedures are communicated to volunteer mentors (Keller, 2005a; Keller \& DuBois, 2019; Tseng \& Seidman, 2007). Program staff implement practices that support volunteers through every stage of program participation, including recruitment, screening, training, matching with a mentee, monitoring and supporting of the match, and match closure (Keller, 2005b). Practice standards for the field, based on evidence from research and practice, are compiled in the Elements of Effective Practice for Mentoring (EEPM; Garringer et al., 2015). Programs employing a greater number of recommended and empirically supported practices are more likely to achieve longer-lasting mentoring relationships and better outcomes for youth mentees (DuBois et al., 2002; Kupersmidt et al., 2017). Likewise, mentor perceptions of structure and support provided by program staff are positively associated with ratings of mentoring relationship quality and with relationship duration (Marshall et al., 2016; Sass \& Karcher, 2013; Weiler et al., 2019) as well as youth outcomes (Herrera et al., 2008). Consistent with principles for volunteer management, these findings suggest the importance of high-quality supervision of mentors by program staff.

Nevertheless, very little research has examined the relationship between program staff and mentors, particularly factors that may affect the quality of supervision and support provided to mentors (Keller, 2007; Marshall et al, 2016; Spencer et al., 2020). Likewise, very little attention has been devoted to how implementation of specific program practices may influence the nature of the volunteer mentor experience (see Herrera et al., 2013; Kupersmidt et al., 2017). One practice that is fundamental to providing ongoing support and supervision to volunteer mentors is engaging in regular contact with them to monitor the progress of the match and 


\section{Support Contacts for Volunteer Mentors}

offer guidance, advice, encouragement, and appreciation (Keller, 2005b). In fact, one study found that regular support calls from staff to mentors were associated with longer-lasting matches and more frequent meetings between mentors and youth (Herrera et al., 2013).

The monitoring and support standards for EEPM recommend that the program contact match participants at least once a month with the following aims (Garringer et al., 2015, p. 61):

- Ask mentors about mentoring activities, mentee outcomes, child safety issues, quality of the mentoring relationship, and impact of mentoring on mentor and mentee.

- Elicit in-depth assessment from mentors and mentees about the quality of their mentoring relationships.

- Provide mentors with access to relevant resources and help mentors address challenges in their mentoring relationships as they arise.

- Provide mentors with feedback on a regular basis regarding their mentees' outcomes and the impact of mentoring on their mentees to continuously improve mentee outcomes and encourage mentor retention.

Accomplishing these aims and providing volunteer supervision that mentors find helpful would seem to require that staff spend some minimum amount of time interacting with the mentor. In this study, we investigated variation in the amount of time mentors reported being devoted to match support contacts. We hypothesized that the length of time reported for staff support contacts would be associated with mentor perceptions regarding the quality of supervision and support received from staff. In addition, we hypothesized that longer support contacts would be associated with other aspects of the volunteer experience, specifically perceptions of a positive organizational climate and feelings of having a positive experience as a volunteer. We also expected these associations would hold after controlling for mentor reports regarding the helpfulness of support contacts and how often the mentor reported using advice suggested by staff during contacts.

\section{Method}

\section{Procedure}

The data are from a multi-state randomized trial evaluating the implementation and impacts of the Quality Mentoring System (QMS), an initiative of MENTOR/The National Mentoring Partnership (Keller et al., 2019). In QMS, MENTOR's state-level affiliates implement a quality rating and improvement strategy with mentoring programs to strengthen their organizational functioning and program service delivery. MENTOR identified eight affiliates to participate in the 


\section{Support Contacts for Volunteer Mentors}

trial. Each affiliate was responsible for recruiting mentoring programs from within their service networks to implement QMS. Affiliates were encouraged to target programs reflecting the diversity of mentoring programs in their area, with attention to factors such as program structure, model, setting, and size. At the outset of the study, baseline data were collected at the agency, staff, and mentor levels. The goal was to obtain baseline surveys from a random sample of 15 mentors from each program, or all mentors if fewer than 15 were active in a program. Program staff produced a numbered list of all eligible mentors in their programs, providing the researchers with the total number and no identifying information. Researchers then generated a random sequence of 20 numbers to indicate the mentors to be sampled. The first 15 mentors were contacted by mentoring program staff who described the study and sought permission from the mentor to release contact information to the research team. If a mentor was not willing or could not be reached, the next prospective participant on the list was contacted until 15 mentors had given permission. Interested mentors were sent an introductory email with information about the study and a link to the online consent form and survey. Participants were offered a $\$ 20$ gift card honorarium. All recruitment and data collection followed IRB-approved procedures. The current study examines only data from volunteer mentor surveys at baseline.

\section{Programs}

A total of 55 youth mentoring programs had volunteer mentors participating in the current study. The number of mentors from each program ranged from 1 to $15(M=10.0, S D=3.33)$. As shown in Table 1, the represented mentoring programs were diverse in terms of their size and their organizational auspices.

Table 1. Characteristics of Mentoring Programs $(N=47)$

\begin{tabular}{|l|c|}
\hline Program Characteristics & $\% / M(S D)$ \\
\hline Organizational structure & \\
\hline Embedded in community organization & $42.5 \%$ \\
Stand-alone non-profit & $29.8 \%$ \\
Other & $27.7 \%$ \\
\hline Affiliated with a national organization & $21.3 \%$ \\
\hline Paid staff devoted to mentoring program & $2.6(3.8)$ \\
\hline Mentors active during last 12 months & $107.6(197.2)$ \\
\hline
\end{tabular}

Note. Volunteer mentors from 55 mentoring programs participated in the study, but program-level surveys were not completed by 8 programs. 


\section{Participants}

A total of 593 mentors from 55 mentoring programs consented to the baseline survey. There were 71 mentors who did not respond to any of the items included in the analysis. In addition, given the focus on volunteers, 18 paid mentors were removed from the analysis. Thus, a total of 504 mentors were included in the final sample for analysis. Table 2 presents descriptive information about the mentors and their mentoring contexts.

Table 2. Participant Demographics and Mentoring Context $(N=504)$

\begin{tabular}{|l|c|c|c|}
\hline $\begin{array}{l}\text { Participant } \\
\text { demographics }\end{array}$ & $\%$ & Mentoring Context \\
\hline $\begin{array}{l}\text { Gender } \\
\text { Female }\end{array}$ & $66.6 \%$ & $\begin{array}{c}\text { Mentoring Model a } \\
\text { One-to-one }\end{array}$ \\
$\begin{array}{l}\text { Race/Ethnicity } \\
\text { White }\end{array}$ & Group & $87.7 \%$ \\
African American & $67.3 \%$ & Team & $6.2 \%$ \\
Asian American & $19.4 \%$ & Multiple mentoring & $5.4 \%$ \\
Hispanic & $5.2 \%$ & Setting & $0.8 \%$ \\
Multiethnic/other & $2.4 \%$ & Community-based & $36.1 \%$ \\
& $5.8 \%$ & School-based & $35.3 \%$ \\
Mean age (SD) & & Site-based & $16.5 \%$ \\
\hline
\end{tabular}

a One-to-one: one mentor, one mentee. Group: one mentor, group of mentees. Team: team of mentors, multiple mentees. Multiple mentoring: multiple mentors, one mentee.

\section{Measures}

Independent Variable

Staff support contact time was measured by asking the mentor, "When agency staff called or met with you to see how your mentoring relationship was going, how long did these conversations typically last?" Response options were no contact, 1-5 minutes, 6-10 minutes, 1120 minutes, and more than 20 minutes. 


\section{Support Contacts for Volunteer Mentors}

\section{Dependent Variables}

Mentor perceptions of program supervision were measured using an adapted version of the Supervisory Relationship Questionnaire (SRQ; Palomo et al., 2010). The SRQ originally was developed to assess relationships between clinical psychology trainees and their supervisors, as reported by the trainee. Four SRQ subscales were adapted to reflect interactions between mentor and agency worker during match support contacts. The structure of contacts was measured using five items (e.g., "My match support sessions/calls are focused."). Whether the mentor saw the worker as a "safe base" was measured using 13 items (e.g., "I feel able to discuss my concerns with my agency worker openly."). The agency worker's commitment to supporting the mentor was measured in 10 items (e.g., "My agency worker appears interested in my development as a volunteer."). Whether the mentor saw the agency worker as a role model was measured with 4 items (e.g., "I respect my agency worker as a professional."). All items had a 6-point scale from strongly disagree to strongly agree. An overall supervision score was computed as a mean of items from all four subscales. This scale had high internal consistency (Cronbach's alpha $=.95)$.

Mentor perceptions of the mentoring agency's organizational culture were assessed with adapted Climate \& Morale and Information Flow subscales of the Organizational Culture Scale (Glaser et al., 1987). Specifically, this measure was adapted so that mentors reported on their experience of the organizational culture from the vantage of their volunteer role. The Climate \& Morale subscale included five items (e.g., "This organization motivates me to give my best efforts."). The Information Flow subscale included four items (e.g., "I know what's happening in the mentoring program."). All items used a 6-point scale from strongly disagree to strongly agree. An overall organizational culture score was computed as a mean of items from the two subscales (Cronbach's alpha $=.95)$.

Mentor service experience was measured using the Organizational Support and Making a Difference subscales of the Inventory of Service Experience (Taylor \& Pancer, 2007). The Organizational Support subscale included six items (e.g., "There is a true sense of teamwork and camaraderie at the organization with which I volunteer."). The Making a Difference subscale included seven items (e.g., "I feel that my volunteer work helps to make a difference.") on a 6-point scale from strongly disagree to strongly agree. An overall service experience score was computed as a mean of items from the two subscales (Cronbach's alpha $=.88)$. 


\section{Support Contacts for Volunteer Mentors}

\section{Control Variables}

To isolate the effects of support contact time, mentor perceptions regarding the value of the support contacts were used as control variables. The mentor's perceptions of the helpfulness of support contacts were measured with two questions from previous research (Herrera et al., 2013). One asked the mentor: "To what extent were your contacts with agency staff helpful in strengthening your mentoring relationship?" Response options were not at all helpful, somewhat helpful, fairly helpful, and very helpful. Mentors also responded to the question: "When you interacted with your mentee or did things for him/her, how often did you use tips or pointers that program staff had suggested to you?" Response options were staff never gave me tips or pointers, staff gave me tips or pointers, but I never used them; very rarely, every few times we met, almost every time we met, and every time we met.

\section{Analysis}

Preliminary analyses examined bivariate associations among the variables in the analyses, including participant demographic characteristics (age, gender, race/ethnicity), program factors (model, setting), and the control variables regarding the helpfulness of support contacts (which were used as categorical variables). Factorial analysis of variance (ANOVA) was used to evaluate the association of staff contact time with mentor-reported quality of program supervision, organizational culture, and service experience. Factorial ANOVA treats independent variables as categorical, allowing for the potential of a nonlinear association between independent and dependent variables. In addition, factorial ANOVA allowed the analysis to account for program-level effects and the nested structure of mentors within mentoring programs. Each hypothesis was tested first with only program affiliation as a control variable and then with helpfulness of support contacts and frequency of using staff advice as control variables. As warranted, post hoc analyses with Bonferroni correction were used to identify and interpret patterns in the results.

\section{Results}

Mentors most frequently reported the amount of time for staff contacts was 6-10 minutes (31.3\%), followed by 1-5 minutes (25.7\%), 11-20 minutes (19.2\%), more than 20 minutes $(12.6 \%)$, and lastly no contact (11.2\%). Statistically significant associations were observed between staff contact time and mentor reports of the helpfulness of staff contacts and how often they used staff advice. Mentors with longer staff contact times reported the contacts were 


\section{Support Contacts for Volunteer Mentors}

more helpful $\left(\mathrm{X}^{2}(12)=115.98, p<.001\right.$, Cramer's $\left.V=.28\right)$ and that they more often used advice suggested by staff $\left(\mathrm{X}^{2}(20)=124.87, p<.001\right.$, Cramer's $\left.V=.25\right)$. Due to these associations, both control variables were retained. As shown in Table 3, there were strong, positive correlations among the three dependent variables: mentor reports of supervision, organizational culture, and volunteer service experience. There were no statistically significant associations between staff contact time and mentor demographic characteristics (age, gender, race/ethnicity), so these variables were not included in further analyses. Nor did mentoring program model (one-to-one vs. others) have a statistically significant association with staff contact time. Program setting was associated with staff contact time $\left(x^{2}(8)=35.56, p<.001\right.$, Cramer's $V=.18)$, with school-based mentors generally reporting less contact. Mentoring model and program setting were not associated with any dependent variables and thus not used as control variables. However, the analyses did include program affiliation to account for program-level effects.

Table 3. Bivariate Correlations Among Dependent Variables

\begin{tabular}{|l|c|c|c|}
\hline & 1 & 2 & 3 \\
\hline 1. Supervision quality & - & & \\
\hline 2. Organizational culture & $.74 * *$ & - & \\
\hline 3. Service experience & $.62^{* *}$ & $.74 * *$ & - \\
\hline Respondents $(M)$ & 499 & 495 & 496 \\
\hline Mean $(S D)$ & $5.28(.62)$ & $5.18(.74)$ & $5.07(.66)$ \\
\hline
\end{tabular}

$* p<.05, * * p<.01$

Evaluation of the primary hypotheses revealed non-linear associations between staff contact time and mentor-reported quality of program supervision, organizational culture, and volunteer service experience. Figure 1 illustrates the overall trend between staff contact time and mentorreported program experiences using overall mean scores of mentor-reported program experiences. 
Figure 1. Mean Ratings of Mentor Volunteer Experiences by Staff Support Contact Time

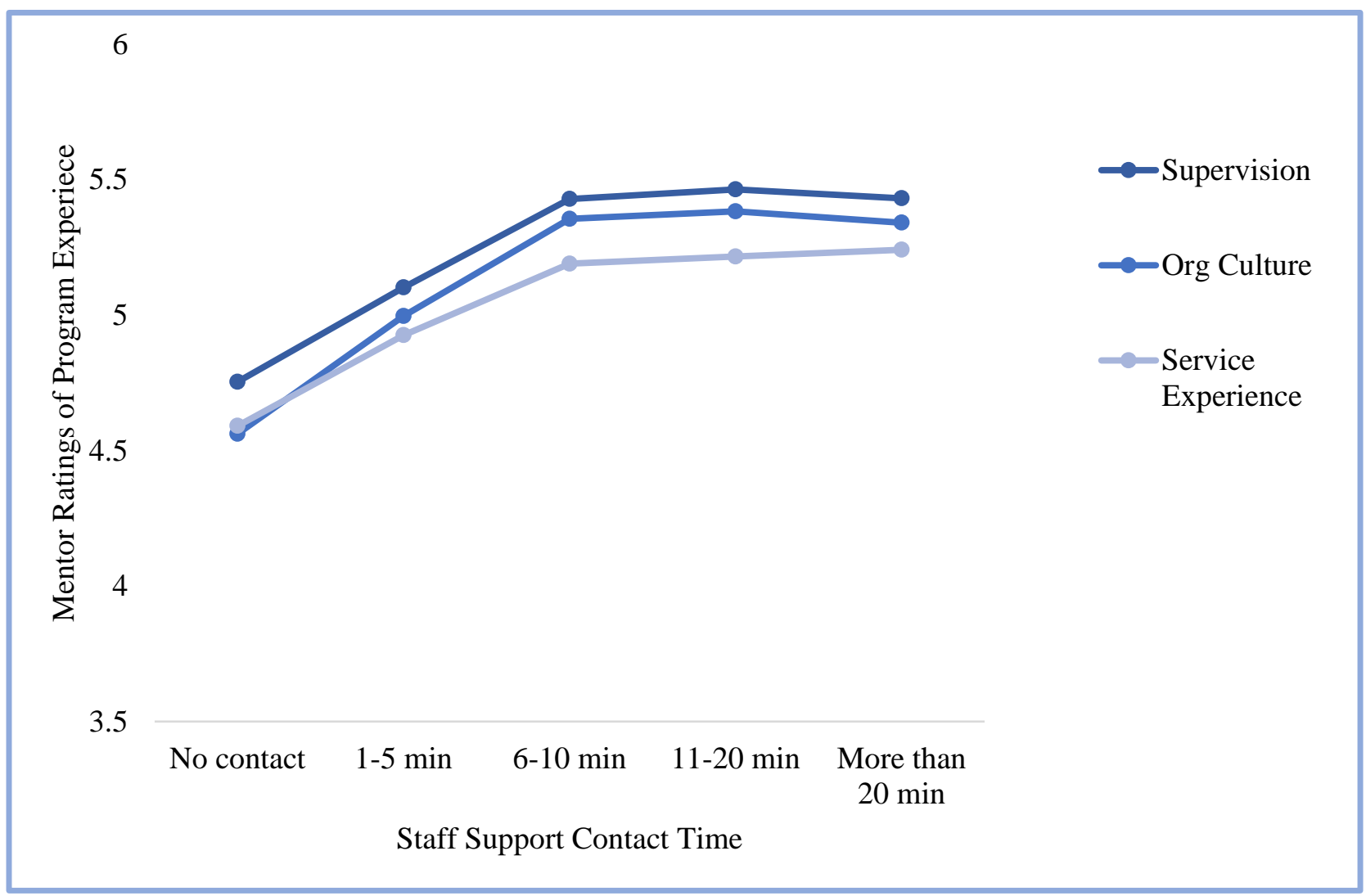

Factorial ANOVA results accounting for the nesting of mentors within programs indicated statistically significant associations between staff contact time and mentor perceptions of program supervision quality $\left(F(4,493)=14.75, p<.001\right.$, partial $\left.\eta^{2}=.12\right)$. Post hoc analyses indicated that mentors who reported no contact time with staff gave lower ratings of program supervision quality compared to mentors who reported any amount of time spent in support contacts. Mentors who typically had 1 to 5 minutes of staff contact also reported lower ratings of program supervision quality compared to mentors with 6 or more minutes of contact time. However, there was a threshold effect, with no statistically significant differences in mentor ratings of program supervision quality between those receiving 6 to10, 11 to 20 , or 20+ minutes of staff contact time. The association between contact time and mentor ratings of program supervision remained when controlling for helpfulness of staff contacts $(F(4,485)=$ $3.85, p<.01)$ and frequency of using staff advice $(F(4,490)=8.34, p<.001)$.

Similarly, a statistically significant association was found between staff contact time and mentor perceptions of organizational culture $\left(F(4,492)=11.51, p<.001\right.$, partial $\left.\eta^{2}=.10\right)$. Post hoc 


\section{Support Contacts for Volunteer Mentors}

analyses indicated that mentors who reported no contact time with staff had the least positive perceptions agency organizational culture. Mentors who typically had 1 to 5 minutes of staff contact also reported fewer positive perceptions of organizational culture compared to mentors with 6 or more minutes of contact time. Again, there were no statistically significant differences in mentor perceptions of organizational culture between those receiving 6 to 10,11 to 20, or $20+$ minutes of staff contact time. The associations between contact time and mentor perceptions of organizational culture remained when controlling for helpfulness of staff contacts $(F(4,483)=2.88, p<.05)$ and frequency of using staff advice $(F(4,488)=7.05, p<.001)$.

Finally, analyses indicated a statistically significant association between contact time and mentor ratings of service experience $\left(F(4,493)=10.26, p<.001\right.$, partial $\left.\eta^{2}=.09\right)$. Post hoc analyses indicated that mentors who reported no contact time with staff had the lowest ratings of service experience. Mentors who typically had 1 to 5 minutes of staff contact also gave lower ratings of service experience compared to mentors with 6 or more minutes of contact time. The threshold effect was observed again, with no statistically significant differences in mentor perceptions of their service experience between those receiving 6 to 10,11 to 20, or 20+ minutes of staff contact time. The association between contact time and mentor perceptions of service experience remained statistically significant when controlling for frequency of using staff advice $(F(4,489))=5.65, p<.001)$, but the association was no longer statistically significant when controlling for helpfulness of staff contacts $(F(4,484)=2.16, p=.07)$.

\section{Discussion}

The findings of this study highlight the importance of match support contacts in shaping the volunteer mentor experience (Keller, 2005b). Mentors who do not have support contacts with program staff give the lowest ratings regarding the quality of volunteer supervision, are the least likely to report the agency has a positive organizational culture, and are the least satisfied with their volunteer service experiences. Among mentors who receive staff support contacts, the amount of time devoted to them seems to matter. Mentors who regularly have contacts longer than 6 minutes rate their supervision more positively, report a more positive organizational culture, and are more pleased with their service experiences than mentors who typically have brief support contacts lasting only 1 to 5 minutes.

During longer support contacts, mentors may be receiving more access to resources to support their mentees, more help addressing challenges in their relationships, and more feedback 


\section{Support Contacts for Volunteer Mentors}

regarding the impact of their relationships on their mentees (Garringer et al., 2015). These longer contacts may help mentors feel they are being closely monitored and provided with the information and support they need to be successful. In fact, the analysis also revealed that longer support contacts are positively associated with mentor perceptions of the overall helpfulness of support contacts and the frequency with which mentors actually use advice suggested by their staff workers. Prior research suggests that mentors who rate program support positively are more likely to be satisfied with their mentoring relationships and to have longer-lasting mentoring relationships (Marshall et al., 2016; Sass \& Karcher, 2013; Weiler et al., 2019). In contrast, very short support contacts may not allow enough time for the program staff person to offer more than surface-level support. While better than no support, short contacts may feel routine or repetitive to mentors, as if the staff person is only making sure the match is still meeting (Spencer et al., 2020).

Of note, there was a non-linear relationship between length of support contacts and mentor perceptions of their volunteer experiences, with a threshold effect above 5 minutes of contact. Specifically, differences on outcome variables were not apparent between mentors who typically had staff support contacts lasting 6 to 10 minutes, 11 to 20 minutes, or more than 20 minutes. Although this finding may suggest limited return on investment for support contacts over 10 minutes, longer contacts may nevertheless be warranted. For example, it may be that the length of the staff support contact is responsive to circumstances in the match. Mentors who struggle with more challenges in their mentoring relationships may spend more time working with staff to find resolutions. A previous study reported longer support contacts for mentors matched to mentees who contended with more individual risk factors (Herrera et al., 2013). While longer contacts might not lead to increases in perceptions of the quality of supervision, organizational culture or service experience, these longer contacts may help mentors stay committed to their mentoring relationships (see Herrera, et al., 2008). Future research should consider mentoring relationship quality and challenges when investigating the length of mentor staff support contacts.

Interpretation of the current findings should consider certain study limitations. For example, the research relied on mentor reports of the typical length of staff support contacts, raising questions regarding the accuracy of recall and the potential for bias based on how mentors experienced those contacts and their experiences with the agency more generally. As noted, mentor ratings regarding the overall helpfulness of support contacts and the frequency of using advice suggested during support contacts were included as control variables and account for 


\section{Support Contacts for Volunteer Mentors}

the possibility that retrospective reports of contact length could be influenced by whether the contacts were considered useful. Likewise, these control variables help to address the possibility that mentors with positive feelings about their volunteer supervision and volunteer experience might assume their support contacts had been of sufficient length. However, the effects of support contact time on the outcomes remained statistically significant when accounting for the control variables, except that controlling for the helpfulness of staff contacts diminished the association between contact time and service experience $(p=.07)$. In general, therefore, the analyses suggested that support contact time had a unique effect on volunteer outcomes over and above the perceived value of the contacts. Nevertheless, future research could employ prospective study designs and include staff-reported contact length or more objective measures such as phone records to triangulate mentor reports.

It is also important to note that this study examined the length of staff support contacts, but data were not available regarding the frequency or the content of the contacts. The EEPM recommends that programs have support contacts with mentors at least monthly, with more frequent contacts if needed (Garringer et al., 2015). It is likely the frequency of contacts also influences volunteer experiences. For example, one study found the frequency of support contacts was associated with longer-lasting matches and more meetings between mentors and youth (Herrera et al., 2013). Therefore, future research should include indicators of both the frequency and duration of support contacts to determine overall dosage of match support. In addition, longitudinal designs could investigate how changes in the frequency and length of support contacts are related to changes in mentor perceptions of their volunteer experience. Likewise, future research could investigate whether the findings reported here hold with more precise measurement of time (e.g., actual minutes) as well as volunteer outcomes. It is possible that a ceiling effect may have prevented further distinctions at higher levels of the outcome measures. Finally, future research could assess how the content of support contacts influences mentor volunteer experiences (Marshall et al., 2016).

Despite the limitations of the current study, the findings reinforce the importance of program staff support contacts with mentors. Mentors who have support contacts report higher levels of program supervision, more positive organizational culture, and better volunteer service experiences compared to those who have no support contacts. Further, the length of these contacts matters. As recommended in the EEPM, support contacts between staff and mentors should be substantive and provide opportunities for staff to learn what is happening in the match and provide resources and guidance to help mentors feel successful (Garringer et al., 


\section{Support Contacts for Volunteer Mentors}

2015). It is likely that mentors with relatively short support contacts do not have the time for these rich conversations, which results in their reporting less satisfaction with their volunteer experiences. While the current study does not specifically address the content of support contacts, programs may begin by monitoring the length of match support contacts and encouraging staff to spend at least 6 to 10 minutes having substantive conversations with mentors. This recommendation may require substantial changes to support procedures and resource allocation for programs that have no support procedures in place or that rely on short phone calls or emails for mentor support.

\section{Acknowledgements}

This study was supported by a grant from the William T. Grant Foundation (\#182183) awarded to Thomas Keller, Renée Spencer, Carla Herrera, and Bowen McBeath. The opinions, findings, conclusions, and recommendations expressed in this publication are those of the authors and do not necessarily reflect those of the sponsor. This project was undertaken in collaboration with MENTOR/The National Mentoring Partnership, several of its affiliates, and many youth mentoring programs. Thomas Keller, Carla Herrera, and Renée Spencer serve on the Research Board of MENTOR's National Mentoring Resource Center.

\section{References}

Brudney, J. L., \& Meijs, L. C. P. M. (2014). Models of volunteer management: Professional volunteer program management in social work. Human Service Organizations: Management, Leadership \& Governance, 38, 3, 297-309. https://doi.org/10.1080/23303131.2014.899281

Clary, E. G., Snyder, M., Ridge, R. D., Copeland, J., Stukas, A. A., Haugen, J., \& Miene, P. (1998). Understanding and assessing the motivation of volunteers: A functional approach. Journal of Personality and Social Psychology, 74(6), 1516-1530. https://doi.org/10.1037//0022$\underline{3514.74 .6 .1516}$

DuBois, D. L., Holloway, B. E., Valentine, J. C., \& Cooper, H. (2002). Effectiveness of mentoring programs for youth: A meta-analytic review. American Journal of Community Psychology, 30(2), 157-197. https://doi.org/10.1023/A:1014628810714

Garringer, M., Kupersmidt, J., Rhodes, J., Stetler, R., \& Tai, T. (2015). Elements of effective practice for mentoring ( $4^{\text {th }}$ ed.). MENTOR/The National Mentoring Partnership. 
Journal of Youth Development | http://jyd.pitt.edu/ | Vol. 15 Issue 4 DOI 10.5195/jyd.2020.879 Support Contacts for Volunteer Mentors

Garringer, M., McQuillin, S., McDaniel, H. (2017). Examining youth mentoring services across America: Findings from the 2016 national mentoring program survey. MENTOR/The National Mentoring Partnership.

Glaser, S. R., Zamanou, S., \& Hacker, K. (1987). Measuring and interpreting organizational culture. Management Communication Quarterly, 1(2), 173-198. https://doi.org/10.1177/0893318987001002003

Grossman, J. B., Chan, C. S., Schwartz, S. E., \& Rhodes, J. E. (2012). The test of time in school-based mentoring: The role of relationship duration and re-matching on academic outcomes. American Journal of Community Psychology, 49(1-2), 43-54. https://doi.org/10.1007/s10464-011-9435-0

Grossman, J. B., \& Rhodes, J. E. (2002). The test of time: Predictors and effects of duration in youth mentoring relationships. American Journal of Community Psychology, 30(2), 199-219.

\section{https://doi.org/10.1023/A:1014680827552}

Grube, J. A., \& Piliavin, J. A. (2000). Role identity, organizational experiences, and volunteer performance. Personality and Social Psychology Bulletin, 26(9), 1108-1119. https://doi.org/10.1177/01461672002611007

Haski-Leventhal, D., \& Bargal, D. (2008). The volunteer stages and transitions model: Organizational socialization of volunteers. Human Relations, 61(1), 67-102. https://doi.org/10.1177\%2F0018726707085946

Herrera, C., DuBois, D. L., \& Grossman, J. B. (2013). The role of risk: Mentoring experiences and outcomes for youth with varying risk profiles. MDRC.

Herrera, C., Kauh, T. J., Cooney, S. M., Grossman, J. B., \& McMaken, J. (2008). High school students as mentors. Public/Private Ventures.

Hidalgo, M. C., \& Moreno, P. (2009). Organizational socialization of volunteers: The effect on their intention to remain. Journal of Community Psychology, 37(5), 594-601. https://doi.org/10.1002/jcop.20317

Keller, T. E. (2005a). A systemic model of the youth mentoring intervention. Journal of Primary Prevention, 26(2), 169-188. https://doi.org/10.1007/s10935-005-1850-2

Keller, T. E. (2005b). The stages and development of mentoring relationships. In D.L. DuBois \& M. Karcher (Eds.), Handbook of youth mentoring (pp. 82-99). Sage.

Keller, T. E. (2007). Program staff in youth mentoring programs: Qualifications, training, and retention. In J. Rhodes (Ed.), Research in Action. MENTOR/The National Mentoring Partnership.

Keller, T. E., \& DuBois, D. L. (2019). Influence of program staff on quality of relationships in a community-based youth mentoring program. Annals of the New York Academy of Sciences. https://doi.org/10.1111/nyas.14289 
Journal of Youth Development | http://jyd.pitt.edu/ | Vol. 15 Issue 4 DOI 10.5195/jyd.2020.879 Support Contacts for Volunteer Mentors

Keller, T. E., Spencer, R., Herrera, C., \& McBeath, B. (2019). Changing youth programs and settings: An experimental evaluation of the Quality Mentoring Systems initiative (Final Program Report). , OR: Portland State University.

Kupersmidt, J. B., Stump, K. N., Stetler, R.L., \& Rhodes, J. E. (2017). Mentoring program practices as predictors of match longevity. Journal of Community Psychology, 45, 630-645. https://doi.org/10.1002/jcop.21883

Macduff, N., Netting F. E., \& O'Connor, M. K. (2009). Multiple ways of coordinating volunteers with differing styles of service. Journal of Community Practice, 17, 400-423. https://doi.org/10.1080/10705420903300488

Malinen, S., \& Harju, L. (2017). Volunteer engagement: Exploring the distinction between job and organizational engagement. Voluntas, 28, 69-89. https://doi.org/10.1007/s11266-016-9823-z

Marshall, J. H., Davis, M. C., Lawrence, E. C., Peugh, J. L., \& Toland, M. D. (2016). Mentors' perceived program support scale: Development and initial validation. Journal of Community Psychology, 44(3), 342-357. https://doi.org/10.1002/jcop.21772

Nencini, A., Romaioli, D., \& Meneghini, A. M. (2016). Volunteer motivation and organizational climate: Factors that promote satisfaction and sustained volunteerism in NPOs. Voluntas, 27, 618-639. https://doi.org/10.1007/s11266-015-9593-z

Omoto, A. M., \& Snyder, M. (1995). Sustained helping without obligation: Motivation, longevity of service, and perceived attitude change among AIDS volunteers. Journal of Personality and Social Psychology, 68(4), 671-686. https://psycnet.apa.org/doi/10.1037/0022-3514.68.4.671

Palomo, M., Beinart, H., \& Cooper, M. J. (2010). Development and validation of the Supervisory Relationship Questionnaire (SRQ) in UK trainee clinical psychologists. British Journal of Clinical Psychology, 49, 131-149. https://doi.org/10.1348/014466509X441033

Penner, L. A. (2002). Dispositional and organizational influences on sustained volunteerism: An interactionist perspective. Journal of Social Issues, 58(3), 447-467. https://doi.org/10.1111/1540$\underline{4560.00270}$

Raposa, E. B., Dietz, N., \& Rhodes, J. E. (2017). Trends in volunteer mentoring in the United States: Analysis of a decade of census survey data. American Journal of Community Psychology, 59, 314. https://doi.org/10.1002/ajcp.12117

Rehnborg, S. J. (2009). Strategic volunteer engagement: A guide for nonprofit and public sector leaders. University of Texas, RGK Center for Philanthropy \& Community Service.

Rochester, C. (1999). One size does not fit all: Four models of involving volunteers in voluntary organizations. Voluntary Action, 1(2), 47-59. 
Journal of Youth Development | http://jyd.pitt.edu/ | Vol. 15 Issue 4 DOI 10.5195/jyd.2020.879

Support Contacts for Volunteer Mentors

Sass, D. A., \& Karcher, M. J. (2013). Analyses of the contribution of case managers to mentor support and match outcomes. In Herrera, DuBois \& Grossman (Eds.), The role of risk: Mentoring experiences and outcomes for youth with varying risk profiles (pp. 120-125). MDRC.

Spencer, R., Gowdy, G., Drew, A. L., McCormack, M., \& Keller, T. E. (2020). It takes a village to break up a match: A systemic analysis of endings in formal youth mentoring relationships. Child \& Youth Care Forum, 49(1), 97-120. https://doi.org/10.1007/s10566-019-09520-w

Stukas, A. A., Clary, E. G., \& Snyder, M. (2014). Mentor recruitment and retention. In D.L. Dubois, \& M. K. Karcher (Eds.), Handbook of Youth Mentoring, ( $2^{\text {nd }}$ ed., pp. 397-410). Sage. https://doi.org/10.4135/9781412996907.n27

Taylor, T. P., \& Pancer, S. M. (2007). Community service experiences and commitment to volunteering. Journal of Applied Social Psychology, 372), 320-345. https://doi.org/10.1111/j.00219029.2007.00162.x

Tseng, V., \& Seidman, E. (2007). A systems framework for understanding social settings. American Journal of Community Psychology, 39, 217-228. https://doi.org/10.1007/s10464-007-9101-8

Weiler, L. M., Boat, A. A., Haddock, S. A. (2019). Youth risk and mentoring relationship quality: The moderating effect of program experiences. American Journal of Community Psychology, 63, 1-2, 73-87. https://doi.org/10.1002/ajcp.12304 